
\title{
COMBINED PHOTON SCANNING TUNNELING MICROSCOPE AND
ATOMIC FORCE MICROSCOPE USING SILICON NITRIDE PROBES
}

\author{
M.H.P. MOERS, R.G. TACK, O.F.J. NOORDMAN, \\ F.B. SEGERINK, N.F. v. HULST \& B. BÖLGER. \\ Department of Applied Physics, University of Twente \\ P.O.Box 217, 7500AE Enschede \\ The Netherlands
}

\begin{abstract}
A Photon Scanning Tunneling Microscope is presented in which $\mathrm{Si}_{3} \mathrm{~N}_{4}$ cantilevers are used as optical probes. This method allows close contact scanning without tip destruction. Simultaneous measurement of the optical signal and the deflection of the cantilever permits a comparison between the optical image and the topography. The optical signal as a function of the distance clearly shows the exponential behaviour of the evanescent field. Several structures have been examined, giving a lateral resolution in the optical image of about $40 \mathrm{~nm}$.
\end{abstract}

\section{Introduction.}

Both Scanning Near field Optical Microscopy (SNOM) and Photon Scanning Tunneling Microscopy (PSTM) are refered to as near field optical techniques. They are, however, based on different principles. In SNOM an aperture is scanned over a surface, acting as a sub wavelength sized lightsource and/or detector [1,2,3]. PSTM, on the other hand, takes advantage of the sub-wavelength information contained in the evanescent field, which is present at an interface with a total internal reflection $[4,5,6]$. Both techniques are capable of high resolution optical imaging. Although the principles are different, the techniques suffer from identical problems. The lateral resolution is mainly determined by the size of the probe, and by the probe-sample distance. Both parameters are difficult to minimize. Furthermore, the tips usually are fragile and will be destroyed by a single touch at the surface.

Several feedback mechanisms are used to keep the probe within close distance of the surface. Apart from the 'classical' feedback on tunneling current (SNOM) [7] or optical intensity (PSTM) $[4,5,6]$, feedback on shear force has been introduced $[8,9]$. This method is closely related to the mode of operation of a dynamic atomic force microscope [10].

In our laboratory a slightly different approach has been followed. $\mathrm{Si}_{3} \mathrm{~N}_{4}$ cantilevers are used as optical near field probes [11]. These cantilevers, with integrated pyramidal tips, are commercially available for Atomic Force Microscopy (AFM). Several features make them suitable for near field microscopy. First, they are optically transparent down to $300 \mathrm{~nm}$, with a refractive index of 2.0. Second, they are microfabricated with a tip radius of $30-50 \mathrm{~nm}$. And finally, the low force 


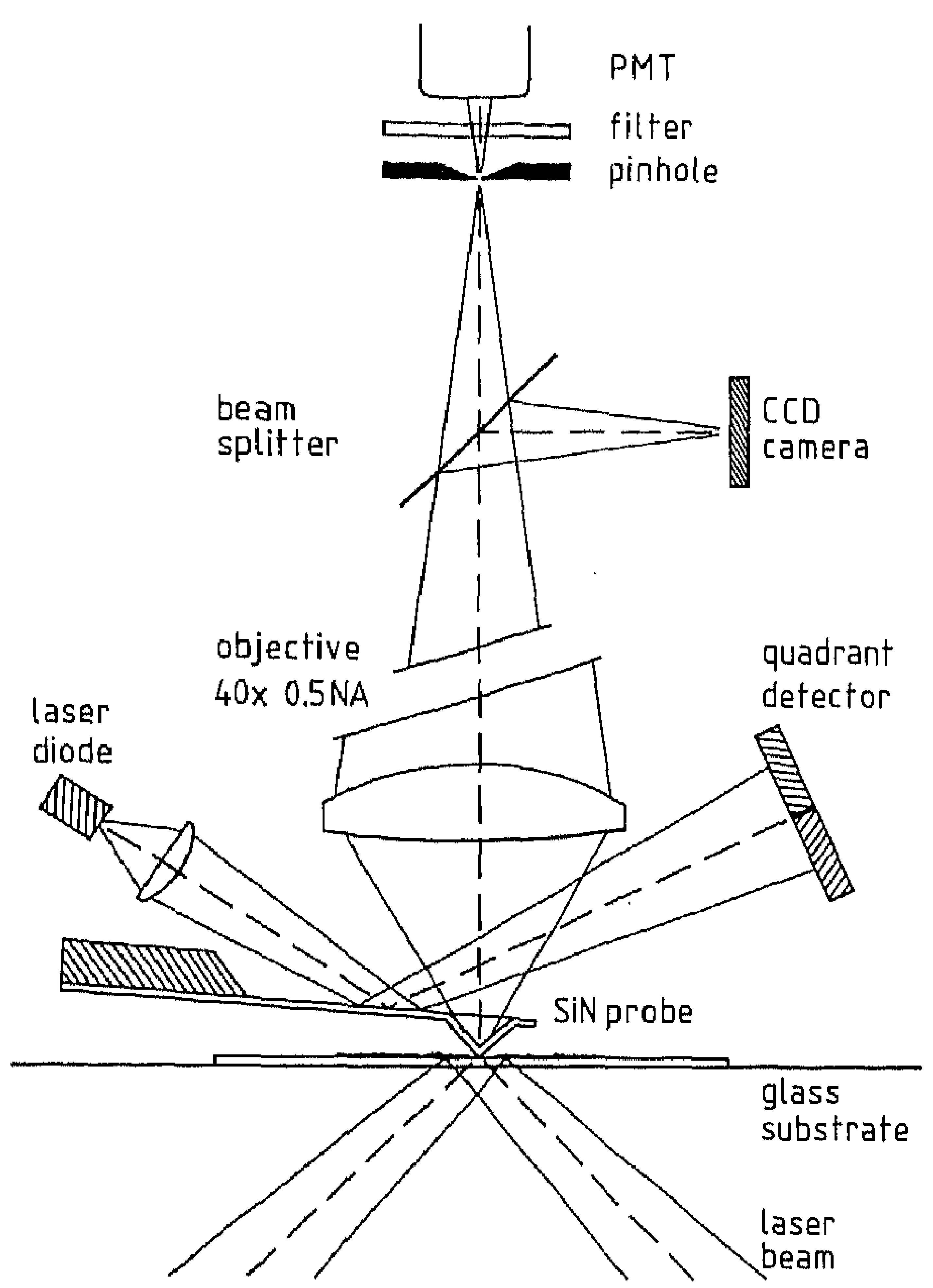

Figure 1. The set-up showing the combination of a PSTM, an AFM and a conventional microscope.

constant of the cantilever allows high speed close-contact scanning of the probe, without destruction of the tip.

The PSTM set-up described in this paper makes use of $\mathrm{Si}_{3} \mathrm{~N}_{4}$ cantilevers to frustrate the evanescent field. An Atomic Force Microscope is incorporated, allowing a simultaneous measurement of the topography. Besides these two scanning probe microscopes the apparatus includes a third, conventional, microscope to preselect the scan area. Results of first measurements on test structures, optical thin films and chromosomes will be shown.

\section{The PSTM set-up.}

The heart of our PSTM set-up is the $\mathrm{Si}_{3} \mathrm{~N}_{4}$ probe (fig.1) [12]. A pyramidal tip (4×4 $\mu \mathrm{m}$ base, $3 \mu \mathrm{m}$ high) is mounted at the end of a long $(200 \mu \mathrm{m})$, thin $(0.6 \mu \mathrm{m})$, Vshaped cantilever (force constant $0.06 \mathrm{~N} / \mathrm{m}$ ). This tip converts the evanescent waves, present above the sample surface, into radiative waves. The evanescent field is 
generated by the total internal reflection of a $5 \mathrm{~mW}$ HeNe laser beam, weakly focused to $100 \mu \mathrm{m}$, on the sample-air interface. The sample is placed on the substrate with index-matching fluid and is raster-scanned by a three axes piezo positioner (Microcontrol). The light, generated by frustrated total internal reflection (FTR), is collected by an extra long working distance objective $(40 \times, N A=0.5,10 \mathrm{~mm})$. A $75 \mu \mathrm{m}$ pinhole in the image plane discriminates between light coming from the apex and light scattered by the sample. The light from the tip is detected by a photomultiplier tube (PMT).

Part of the light is split towards a CCD camera. This allows simultaneous viewing of tip and sample, which is essential for preselecting the scan area [13].

An Atomic Force Microscope is incorporated in the set-up by means of a beam deflection system [14]. A laser diode is focused on the upper side of the cantilever, and the reflected beam is detected by a quadrant detector. Small deflections of the cantilever induce a shift of the laser spot on the detector. Two modes of operation are possible for the AFM. While scanning in open loop, the cantilever follows the topography of the sample, and the deflection is monitored. Alternatively, in the closed loop mode the deflection is preset to a certain value, corresponding to a constant force, with a feedback loop controlling the position of the sample relative to the probe.

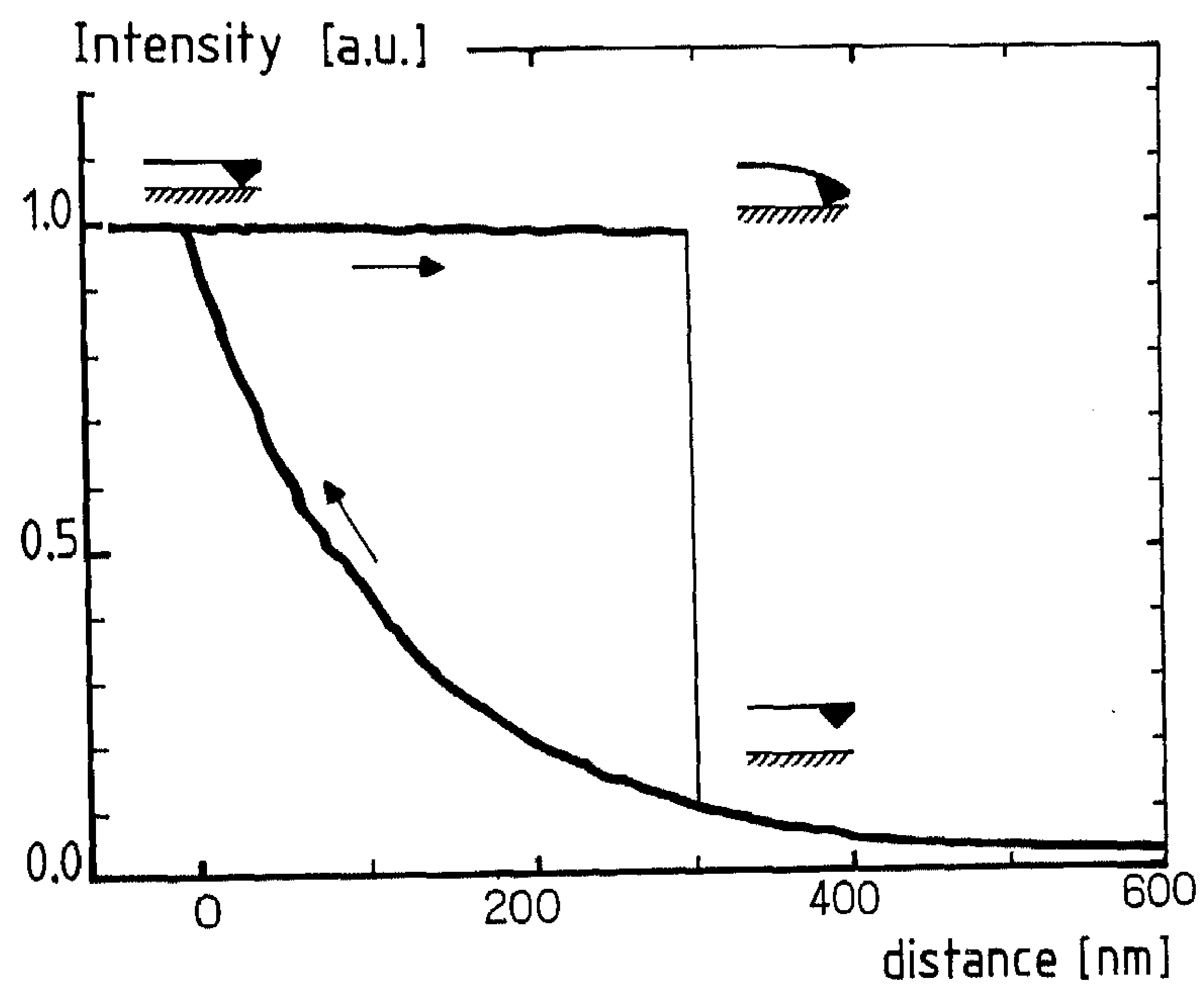

Figure 2. The FTR intensity as a function of the probe-sample distance. On approaching, the exponential behaviour is clearly observed. On retract
the tip sticks to the surface due to adhesive forces of the water film. 


\section{Results and discussion}

Optical signal versus distance curve. The exponential behaviour of the evanescent field [6] is clearly observed when the probe approaches a glass substrate, as shown in figure 2. An intensity level of approximately $0.1 \mathrm{nW}$ is reached when the tip touches the surface. All PSTM images shown in these proceedings have been obtained in this contact regime, with feedback on force. On retracting the tip, contact is maintained due to adhesive forces of the water film on the surface [15]. The cantilever typically bends over $300 \mathrm{~nm}$ (strongly depending on the relative humidity), corresponding to $18 \mathrm{nN}$ adhesion force, until contact is released. This causes a drastic decrease in intensity.

Water film. The effect of the water film is clearly demonstrated on days with a high relative humidity $(>70 \%)$. On these occasions a droplet of liquid, up to $1 \mu \mathrm{m}$ in size, is observed with the conventional microscope at the contact area. The size of this droplet is strongly depending on the relative humidity. Although it does not effect the AFM, the working of the PSTM strongly depends on the humidity. Probably the area of FTR is determined by the size of the water droplet.

Grating. Figure 3 displays a $1 \mu \mathrm{m}$ period symmetric $\mathrm{Si}_{3} \mathrm{~N}_{4}$ grating, with $200 \mathrm{~nm}$ deep tracks. The structure is clearly resolved in the force image, although the low parts seem to be narrower than the high parts, due to the tip convolution problem. The grating is also visible in the PSTM image, albeit with a worse resolution.

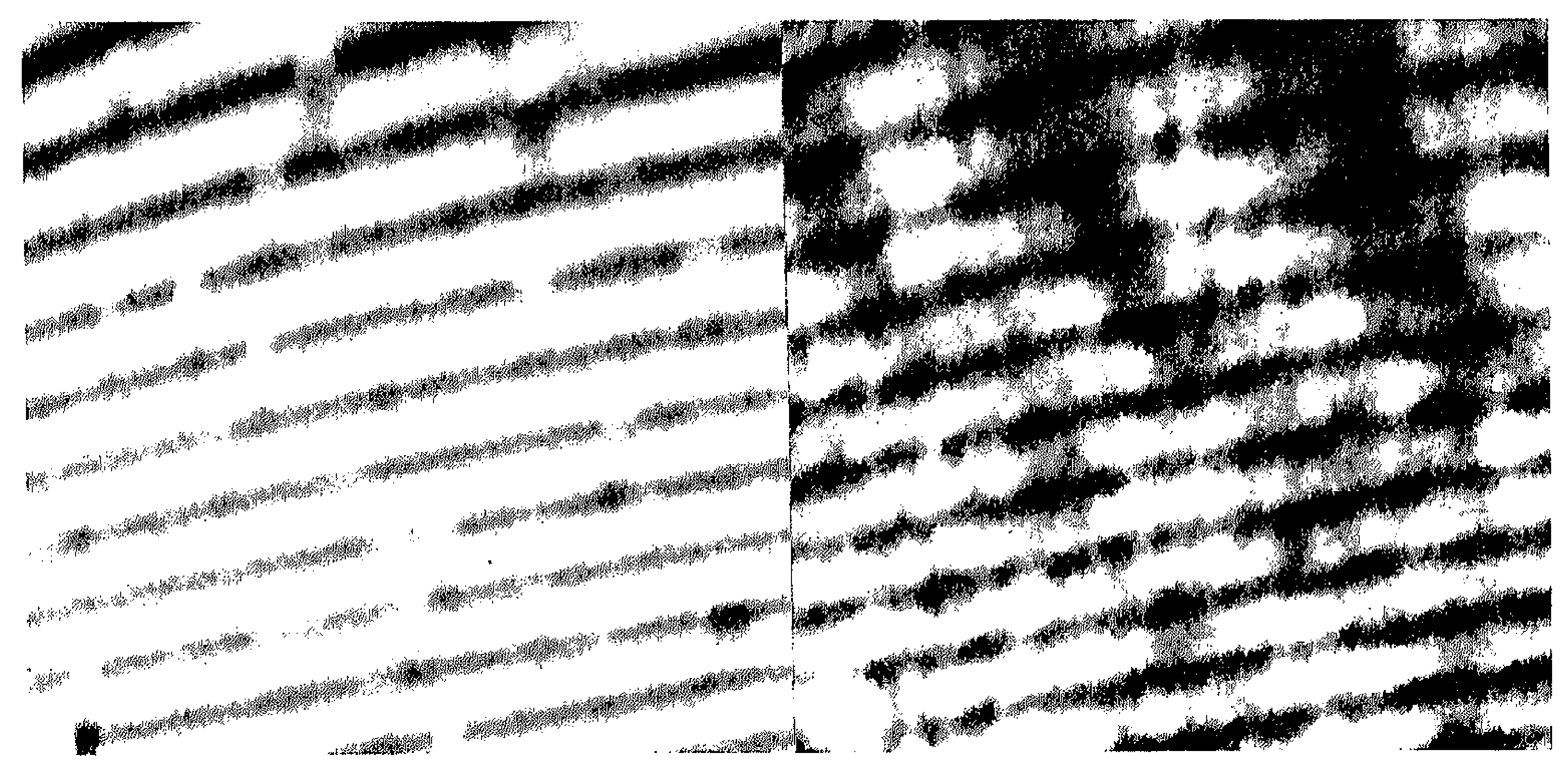

Figure 3. A $11 \times 11 \mu \mathrm{m}$ scan of a $1 \mu \mathrm{m}$ period grating.

a) The AFM image, obtained in closed loop mode.

b) The corresponding PSTM image. 
Chromosome. Preliminary results of biological objects with the PSTM-AFM combination are shown in figure 4. The inclusion of the conventional microscope is essential, especially for finding localized objects like cells and chromosomes. Figure 4a displays the topography of a metaphase chinese hamster lung chromosome. Although biological objects are relatively soft samples, this does not seem to affect the quality of the image. No material is removed over several scans, with forces in the order of $10 \mathrm{nN}$. The corresponding PSTM image shows two fundamental problems of PSTM on structures with strong surface topography. The laser beam is incident from the left. On this side of the chromosome an interference pattern is observed. This is an interference between the incident surface wave and the reflected wave from the chromosome [16]. One should notice that this interference pattern is restricted to the surface, so it can not be seen in the far field. To the right, on the 'shadow' side, radiative waves cause a pattern which is extending for about $4 \mu \mathrm{m}$ beyond the structure, due to the disturbance of the surface wave. Possibly the conditions for total internal reflection are not matched at the downward slope of the chromosome.

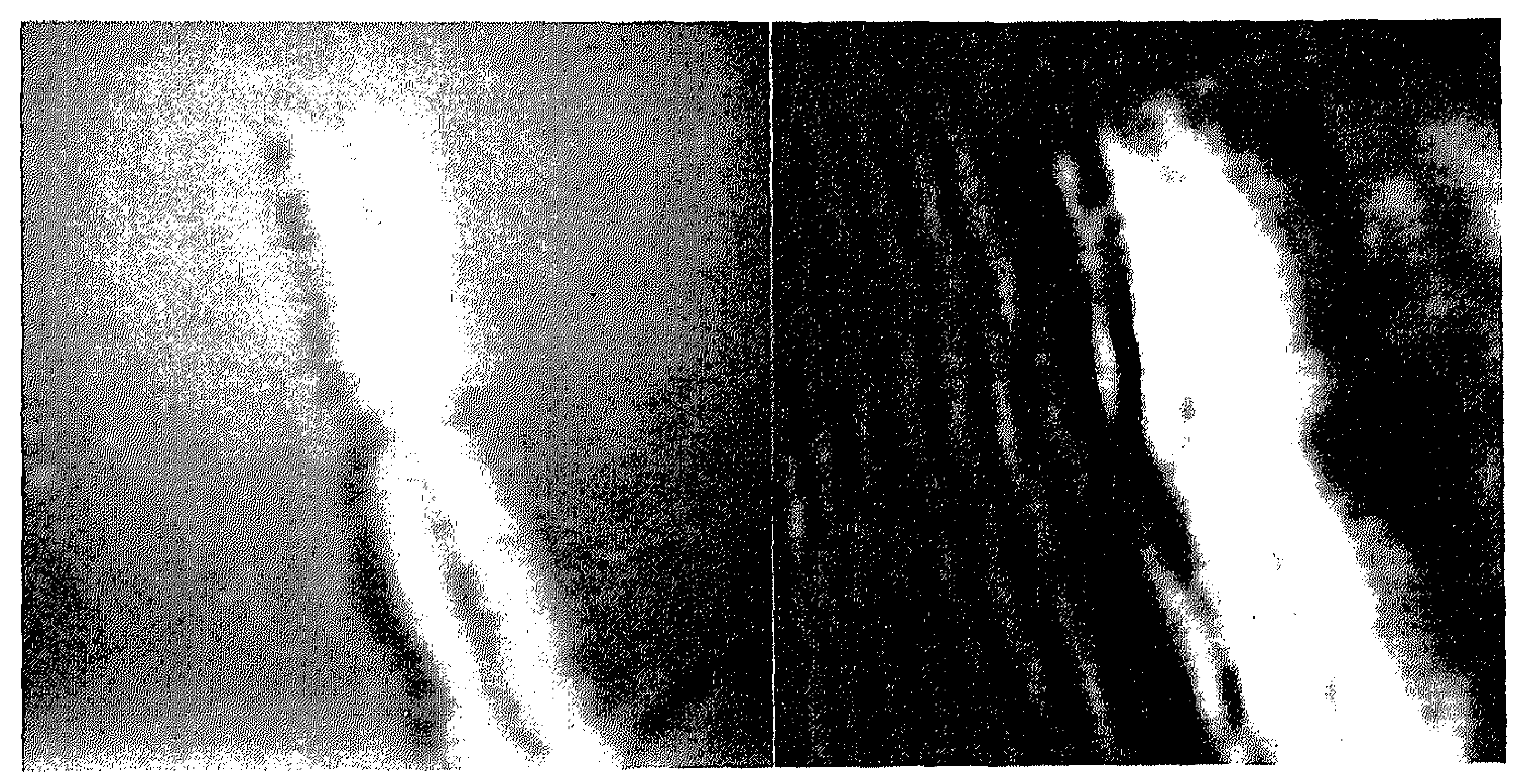

Figure 4. A $15 \times 15 \mu \mathrm{m}$ scan of a chromosome.

a) The AFM image clearly showing the topography.

b) The corresponding PSTM image showing interference and scattering.

Supertips. In order to reduce the contact area between the probe and the surface, the use of 'supertips' can be considered. In a Scanning Electron Microscope (SEM) a thin carbon needle is grown on a $\mathrm{Si}_{3} \mathrm{~N}_{4}$ tip by local electron-beam induced deposition of residuents [17]. This supertip can be $20-100 \mathrm{~nm}$ wide and up to several microns long. The advantage of these tips is threefold. In the first place the coupling between surface and probe is better localized, as can be seen by the size of the 
light spot. In the second place the apex angle is much less than for normal tips, which improves the resolution when imaging steep structures. And finally, the adhesion forces are reduced.

It seems remarkable that FTR is also possible with supertips, because it is not clear whether the carbon structure is optically transparent. The obtained intensity is, however, of the same order of magnitude as with normal $\mathrm{Si}_{3} \mathrm{~N}_{4}$ tips. Of course, any structure placed in the evanescent field will scatter, whatever the material it is made of. Optical transparent material is only necessary to guide the scattered light to the detector (tapered fibers) or to enable imaging with the objective ( $\mathrm{Si}_{3} \mathrm{~N}_{4}$ cantilevers).

Indium Tin Oxide. Figure 5 shows a $1 \times 1 \mu \mathrm{m}$ image of an Indium Tin Oxide film (Baltracon), obtained with a supertip. The granular structure of crystallites, 30$100 \mathrm{~nm}$ in size, is clearly resolved in the force image. In the optical image similar structures are observed, although the grains appear dark. This can be due to the refractive index and limited transmittivity of the crystallites. In the area between the grains the tip couples to the substrate resulting in a rather sharp light rim around the grains. Crystallites, $50 \mathrm{~nm}$ in size, are clearly resolved.

On the other hand, the topography could force the tip out of the evanescent field, affecting the efficiency of coupling the light into the probe. If this is true, one should realize, however, that this is an effect which is inherent to PSTM (in fact, to all scanning probe microscopes). It is not a limitation of the use of $\mathrm{Si}_{3} \mathrm{~N}_{4}$ probes. The lateral resolution of the PSTM image seems to be about $40 \mathrm{~nm}$, better than obtained with normal $\mathrm{Si}_{3} \mathrm{~N}_{4}$ cantilevers.

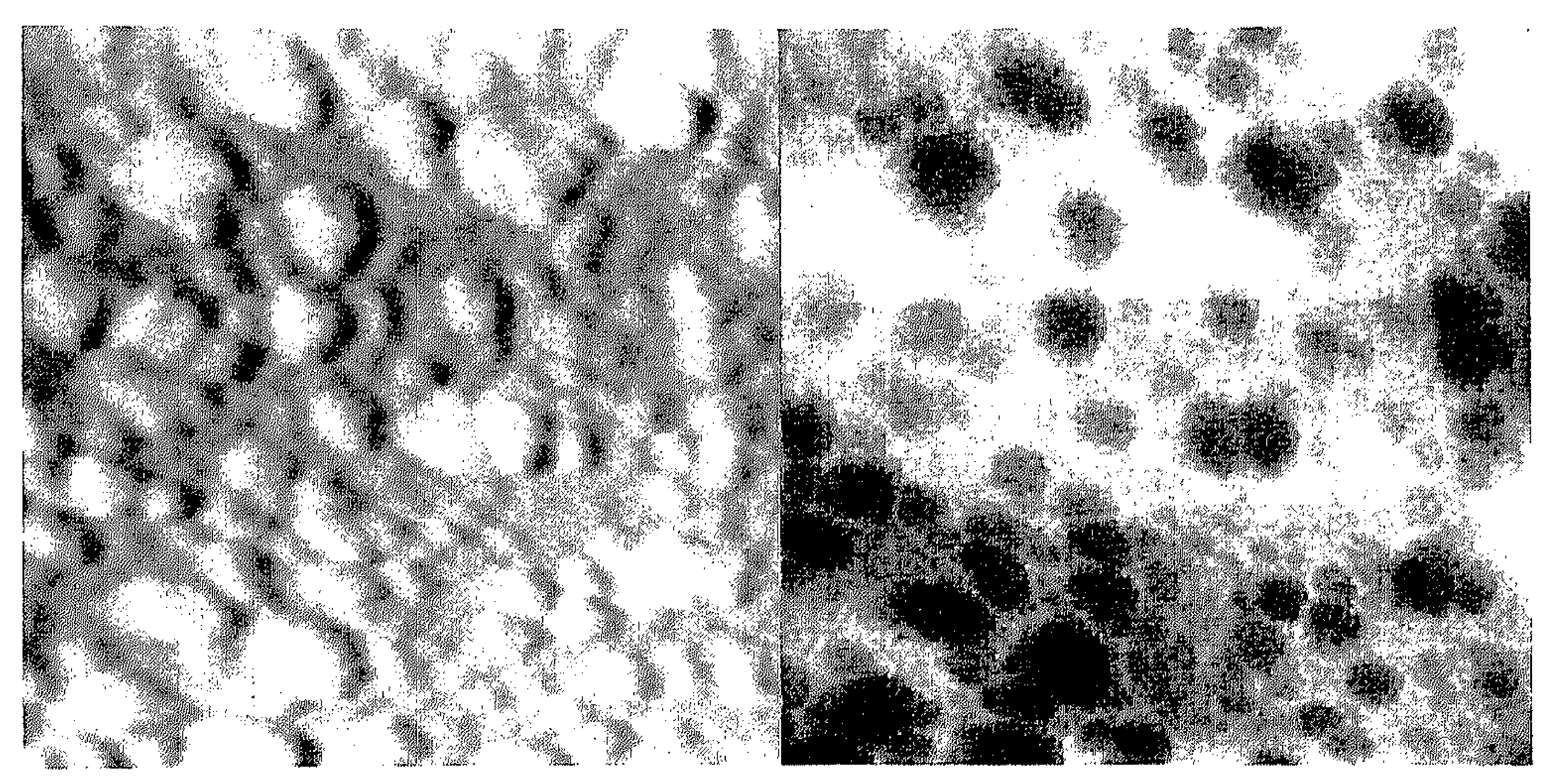

Figure 5. A $1 \times 1 \mu \mathrm{m}$ scan of Indium Tin Oxide.

a) The AFM image showing the granular structure of ITO.

b) The corresponding PSTM image. 


\section{Conclusions}

The operation of a Photon Scanning Tunneling Microscope with a $\mathrm{Si}_{3} \mathrm{~N}_{4}$ cantilever as optical probe has been demonstrated, as well as the problems inherent to the technique. Routine close contact scanning without tip destruction is possible. Simultaneous operation of the Atomic Force Microscope allows comparison between the optical image and topography.

The optical signal versus distance curve indicates that the frustrated evanescent field is detected. Problems arise first then, when a sample is placed on the substrate. Interference and scattering are major problems of Photon Scanning Tunneling Microscopy, as well as the coupling between topography and optical signal. These problems have not completely been solved yet.

Indium Tin Oxide, a granular structure with dimensions well below the optical wavelength, gives good results. With supertips, a resolution in the optical image of about $40 \mathrm{~nm}$ is obtained. The effect of adhesion due to the water film seems to be reduced by the use of supertips.

\section{Acknowledgements}

This work is mainly supported by the Dutch Foundation for Fundamental Research (FOM). The authors wish to thank K. v.d. Werf for his help with the construction of the AFM and B. Otter for the fabrication of the supertips.

\section{References}

1. Pohl, D.W., Denk, W. and Lanz, M. (1984) 'Optical stethoscopy: Image recording with resolution $\lambda / 20^{\prime}$, Appl.Phys.Lett. 44(7), 651-653.

2. Betzig, E., Isaacson, M. and Lewis, A. (1987) 'Collection mode near-field scanning optical microscopy', Appl.Phys.Lett. 51(25), 2088-2090.

3. Betzig, E, Trautman, J.K., Harris, T.D. and Weiner, J.S. (1991) 'Breaking the diffraction barrier: Optical microscopy on a nanometric scale', Science 251, 1468-1470.

4. Reddick, R.C., Warmack, R.J. and Ferrell, T.L. (1989) 'New form of optical microscopy', Phys.Rev. B39(1), 767-770.

5. Courjon, D., Sarayeddine, K. and Spajer, M. (1989) 'Scanning tunneling optical microscopy', Opt.Comm. 71, 23-28.

6. v.Hulst, N.F., de Boer, N.P. and Bölger, B. (1991), 'An evanescent-field optical microscope', J.Micr. 163(2), 117-130.

7. Dürig, U., Pohl, D. and Rohner, F. (1986), 'Near-field optical scanning microscopy with tunnel-distance regulation', IBM J.Res.Develop. 30(5), 478483.

8. Betzig, E., Finn, P.L. and Weiner, J.S. (1992), 'Combined shear force and nearfield scanning optical microscopy', Appl.Phys.Lett. 60, 2484-2486.

9. Toledo-Crow, R., Yang, P.C., Chen, Y. and Vaez-Iravani, M. (1992), 'Near field differential scanning optical microscope with atomic force regulation', App1.Phys.Lett. 60, 2957-2959.

10. Den Boef, A.J. (1991), 'The influence of lateral force in Scanning Force Microscopy', Rev.Sci.Instrum. 62, 88-92.

11. v.Hulst, N.F., Moers, M.H.P., Noordman, O.F.J., Faulkner, T., Segerink, F.B., 
v.d.Werf, K.O., de Grooth, B.G. and Bölger, B. (1992) 'Operation of a scanning near field optical microscope in reflection in combination with a scanning force microscope', SPIE 1639, 36-43.

12. Park Scientific Instruments, Sunnyvale, California, USA.

13. Putman, C.A.J., v.d.Werf, K.O., de Grooth, B.G., v.Hulst, N.F., Segerink, F.B. and Greve, J. (1992) 'Atomic force microscope with integrated optical microscope for biological applications', Rev.Sci.Instrum. 63(3), 1914-1917.

14. Meyer, G. and Amer, N.M. (1988), 'Novel optical approach to atomic force microscopy', Appl.Phys.Lett. 53(24), 2400-2402.

15. Weisenhorn, A.L., Hansma, P.K., Albrecht, T.R. and Quate, C.F. (1989), 'Forces in atomic force microscopy in air and water', Appl.Phys.Lett 54, 2651-2653.

16. v.Hulst, N.F., Segerink, F.B., Achten, F. and Bölger, B. (1992) 'Evanescentfield optical microscopy: effects of polarization, tip shape and radiative waves', Utramicroscopy 42-44, 416-421.

17. Keller, D.J. and Chih-Chung, C. (1992) 'Imaging steep, high structures by scanning force microscopy with electron beam deposited tips', Surf.Sci. 268, 333-339. 